\title{
Applications of Antisense Peptide Nucleic Acid in Biomedicine and Biosciences
}

\author{
Anjali Gupta* and Kavita Khatana \\ Department of Chemistry, Galgotias University, India
}

Submission: October 26, 2017; Published: December 11, 2017

*Corresponding author: Anjali Gupta, Department of Chemistry, School of Basic and Applied Sciences, Galgotias University, Greater Noida, UP, India, Email: anjali21in@gmail.com

\section{Opinion}

Peptide Nucleic Acids (PNAs) are synthetic mimics of DNA/ RNA [1] containing N-2-aminoethylglycine repeating units in place of sugar-phosphate backbone of DNA or RNA. Due to the absence of electrostatic repulsions, PNA/DNA duplexes are thermally more stable as compared to DNA/DNA double helix. The sequence selective nature and mismatch intolerance of PNA probes make them useful in biomedical and biosciences applications [2]. PNAs can be used as antisense agents that block the translation process and inhibit the synthesis of targeted protein. These antisense oligonucleotides are used to treat genetic mutations or different infections.

\section{Anticancer agents}

For instance, antisense PNA (APNA) was found to inhibit the over expression of microRNA-155 which is responsible for causing various diseases especially tumors. An encapsulated APNA conjugate was found to obstruct microRNA-155 over expression that resulted in finding of potential anticancer therapeutics [3]. In another report, radio labeled conjugate of PNA-peptide inhibited the over expression of B-cell leukemia-2, a proto-oncogene in non-Hodgkin's lymphoma via antisense activity [4]. Another citation also concluded the inhibition of CerbB-2 proto-oncogene over expression through ${ }^{125} \mathrm{I}$ labeled APNA in human ovarian cancer cell lines SKOV3 [5]. Furthermore, it was also reported that DNA transcript promoter of lung cancer SPC-A1 was blocked by APNA after changing its physiological characteristics [6]. In addition, APNAs also provide a new methodology which can suppress nonsense terminations that are found to cause cancer and a large number of hereditary genetic disorders [7].

\section{Antiviral agents}

APNA can also be potentially used as antiviral agents and it was evaluated by Lee et al. [8] with PRF-1 signal as target. In viruses, PRF-1 signal causes synthesis of RNA replicase polyproteins for genome replication. Recently, APNA with low cellular toxicity was reported to target replication process of Hepatitis B virus. This virus was found to be responsible for causing chronic hepatitis, cirrhosis and carcinoma [9].

\section{Antibacterial agents and antibiotics}

Nowadays, increasing multidrug resistance in bacteria poses major threat for human health. It is one of the serious problem which is an alarm for the need of new antibacterial drugs as these pathogenic bacteria exhibit resistance for traditional antibiotics due to their excessive use.

APNAs as antibacterial agents were found to treat infections due to resistant reversal in bacteria. APNA was found to be a therapeutic platform that targets varied genes in Pseudomonas aeruginosa that acquired resistance to most of the antibiotics [10]. Acinetobacter baumannii causes common and severe community and hospital acquired infections. In another report, Wang et al. [11] reported APNAs to show strong inhibitory effects in multiresistant drug for Acinetobacter baumannii with minimum bactericidal and inhibitory concentration of 10 and $5 \mu \mathrm{M}$, respectively.

APNAs were also found to effective antibacterial agent and its potential was studied by Sadeghizadeh et al. [12]. The studies revealed that PNA conjugate might prove as an outstanding candidate for immunological and DNA vaccine. These APNAs were also found to target essential genes diseased with Klebsiella pneumoniae $\left(10^{4} \mathrm{CFU}\right)$ with negligible toxicity to the human cells [13].

\section{Conclusion}

PNAs, created in 1991 are the natural nucleic acid analogues which are found to possess potential applications in biomedicine and biosciences due to their stability and high binding affinity. Due to their great impact as antisense agents, PNAs are gaining interest in the field of research and development as anticancer, antiviral and antibacterial agents. 


\section{Current Trends in Biomedical Engineering \& Biosciences}

\section{Acknowledgement}

Author A. Gupta gratefully acknowledges the financial assistance from Science and Engineering Research Board (DST), New Delhi.

\section{References}

1. Nielsen PE, Egholm M, Berg RH, Buchardt O (1991) Sequence-selective recognition of DNA by strand displacement with a thymine-substituted polyamide. Science 254(5037): 1497-1500

2. Kiliszek A, Banaszak K, Dauter Z, Rypniewski W (2015) The first crystal structures of RNA-PNA duplexes and a PNA-PNA duplex containing mismatches-toward antisense therapy against TREDs. Nucleic Acids Res 44(4): 1937-1943.

3. Babar IA, Cheng CJ, Booth CJ, Liang X, Weidhaas JB, et al. (2012) Nanoparticle-based therapy in an in vivo microRNA-155 (miR-155)dependent mouse model of lymphoma. Proc Natl Acad Sci 109(26) E1695-E1704.

4. Balkin ER, Liu D, Jia F, Ruthengael VC, Shaffer SM, et al. (2014) Comparative bio distributions and dosimetry of [177Lu]DOTA-antibcl-2-PNA-Tyr3-octreotate and [177Lu]DOTA-Tyr3-octreotate in a mouse model of B-cell lymphoma/leukemia. Nuclear Medicine and Biology 41(1): 36-42.

5. Huang H-m, Zhang B, Hu J-m (2010) Depressed expression of CerbB-2 mRNA and Her-2 protein in human ovarian cancer Cell line SKOV3 by $\sim(125)$ I labeled antisense peptide nucleic acid. Suzhou Daxue Xuebao Yixueban 30: 1014-1017.

6. Wang GH, Huang GJ, Yu SC, Zhang ZY, Qian GS (2009) The effect of blocking the expression of mtDNA on lung cancer SPC-A1 cell by antisense peptide nucleic acid. Chongqing Yike Daxue Xuebao 34 1457-1461.

7. Kulyte A, Dryselius R, Karlsson J, Good L (2005) Gene selective suppression of nonsense termination using antisense agents. Biochimica et Biophysica Acta-Gene Structure and Expression 1730(3): 165-172.

8. Ahn DG, Lee W, Choi JK, Kim SJ, Plant EP, et al. (2011) Interference of ribosomal frame shifting by antisense peptide nucleic acids suppresses SARS coronavirus replication. Antiviral Res 91(1): 1-10.

9. Zeng Z, Han S, Hong W, Lang Y, Li F, et al. (2016) A tat-conjugated peptide nucleic acid Tat-PNA-DR inhibits hepatitis B virus replication in vitro and in vivo by targeting LTR direct repeats of HBV RNA. Mol Ther Nucleic Acid 5: e295.

10. Maekawa K, Azuma M, Okuno Y, Tsukamoto T, Nishiguchi K, et al. (2015) Antisense peptide nucleic acid-peptide conjugates for functional analyses of genes in pseudomonas aeruginosa. Bioorg Med Chem 23(22): 7234-7239.

11. Wang H, He Y, Xia Y, Wang L, Liang S (2014) Inhibition of gene expression and growth of multidrug-resistant acinetobacter baumannii by antisense peptide nucleic acids. Mol Biol Rep 41(11): 7535-7541.

12. Sadeghizadeh M, Nikravesh A, Behmanesh M, Good L (2009) Cellular morphology and immunologic properties of escherichia coli treated with antimicrobial antisense peptide nucleic acid. Iranian Journal of Pathology 4: 13-18.

13. Kurupati P, Tan KSW, Kumarasinghe G, Poh CL (2007) Inhibition of gene expression and growth by antisense peptide nucleic acids in a multiresistant -lactamase-producing klebsiella pneumoniae strain. Antimicrobial Agents and Chemotherapy 51: 805-811.

This work is licensed under Creative Commons Attribution 4.0 Licens

DOI: 10.19080/CTBEB.2017.10.555789 